\title{
Light Paired with Serotonin in vivo Produces Both Short- and Long-Term Enhancement of Generator Potentials of Identified B-Photoreceptors in Hermissenda
}

\author{
T. Crow and J. Forrester \\ Department of Physiology, University of Pittsburgh School of Medicine, Pittsburgh, Pennsylvania 15261, and Department \\ of Neurobiology and Anatomy, The University of Texas Medical School at Houston, Houston, Texas 77225
}

\begin{abstract}
An in vivo conditioning procedure consisting of light paired with the direct application of 5-HT to the exposed but otherwise intact nervous system of Hermissenda produces a long-term modification of phototactic behavior. The longterm change in phototactic behavior produced by in vivo conditioning is dependent upon pairing light with 5-HT. In this paper, we investigate neural correlates of in vivo conditioning detected in 2 different identified photoreceptors. We found that in vivo conditioning produces a short-term and long-term enhancement of light-evoked generator potentials recorded from medial and lateral B-photoreceptors. We show that short-term enhancement is not dependent upon pairing light with 5-HT, is observed in both lateral and medial B-photoreceptors, and is expressed by a larger peak and plateau phase of light-evoked generator potentials. In contrast to short-term enhancement, we found that long-term enhancement is dependent upon pairing light with $5-\mathrm{HT}$, is detected in only lateral B-photoreceptors, and is expressed by a larger steady-state plateau phase of light-evoked generator potentials. We also present evidence that the direct action of 5-HT interacting with light- and/or voltage-dependent processes is sufficient to mimic the effects of in vivo conditioning on long-term enhancement. These results suggest that long-term enhancement may contribute to modified phototactic behavior in Hermissenda produced by 1-trial in vivo conditioning.
\end{abstract}

Phototaxis in Hermissenda, an example of visually guided behavior, can be modified by a 1-trial in vivo conditioning procedure. The conditioning trial consists of pairing light, the conditioned stimulus (CS), with the direct application of 5-IIT to the exposed circumesophageal nervous system of otherwise intact Hermissenda (Crow and Forrester, 1986). While the cellular mechanisms for the modification of behavior are not known, it has been proposed that the effect of in vivo conditioning on phototactic behavior may be due, in part, to the action of 5-HT interacting with light- and/or voltage-dependent conductances in identified B-photoreceptors (Crow, 1988). In sensory systems

\footnotetext{
Received May 22, 1990; revised Oct. 9, 1990; accepted Oct. 17, 1990.

This work was supported by National Institute of Mental Health Grant MH40860. We thank Diane Galler for typing the manuscript and Tracy Hodgson for reading an earlier draft of the manuscript.

Correspondence should be addressed to Dr. T. Crow, Department of Neurobiology and Anatomy. The University of Texas Medical School at Houston, Houston, TX 77225.

Copyright (C) 1991 Society for Neuroscience 0270-6474/91/110608-10\$03.00/0
}

of a number of invertebrate species, neuromodulators such as 5-HT have pronounced effects on both behavior and the excitability of identified target neurons (Adolph and Tuan, 1972; Barlow et al., 1977; Gershon, 1977; Corrent et al., 1978; Klein and Kandel, 1978; Gelperin, 1981; Mackey and Carew, 1983; Crow and Bridge, 1985; Jacklet and Acosta-Urquidi, 1985; Ocorr and Byrne, 1985; Acosta-Urquidi et al., 1989; Dixon and Atwood, 1989; Farley and Wu, 1989; Hawkins, 1989; Hawkins and Schacher, 1989; Mackey et al., 1989). Application of 5-HT to the visual system of Hermissenda enhances both the amplitude and the duration of light-evoked generator potentials recorded from type-B photoreceptors (Crow and Bridge, 1985; Farley and Auerbach, 1986; Sakakibara et al., 1987). Biochemical studies have demonstrated that $5-\mathrm{HT}$ is endogenous to the cerebropleural and pedal ganglia of Hermissenda (Heldman and Alkon, 1978). Several serotonergic neurons that may provide a presynaptic source of serotonergic input to the visual system were identified in a recent immunohistochemical study of the circumesophageal nervous system. These results revealed 5-HTimmunoreactive fibers and varicosities in close proximity to the optic nerve and in the synaptic region in the neuropil near the photoreceptor synaptic terminals (Land and Crow, 1985).

In this paper, we have examined intrinsic modifications of light-evoked generator potentials recorded from identified B-photoreceptors following in vivo conditioning. We have found that in vivo conditioning produces both a short-term and a longterm enhancement of light-evoked generator potentials recorded from different identified type-B photoreceptors. Short-term enhancement examined $1 \mathrm{hr}$ after conditioning was found in both medial and lateral B-photoreceptors and was not dependent upon pairing the CS with 5-HT. Long-term enhancement examined $24 \mathrm{hr}$ after in vivo conditioning was found only in lateral B-photoreceptors and was dependent upon pairing the CS with 5-HT. We present evidence that long-term enhancement is expressed in lateral B-photoreceptors by a larger amplitude of the steady-state plateau phase of the generator potential without affecting the amplitude of the transient peak.

\section{Materials and Methods}

Animals. A total of 223 adult Hermissenda crassicornis was used in the experiments. Hermissenda were obtained from Sea Life Supply Co. (Sand City, CA). The animals were maintained in an artificial seawater (ASW) aquarium at $14 \pm 0.5^{\circ} \mathrm{C}$ on a $12-\mathrm{hr}$ light/12-hr dark cycle. Animals were fed small pieces of scallops each day during the experiments.

Surgery. Animals were anesthetized by a $0.25-0.5-\mathrm{ml}$ injection of isotonic $\mathrm{MgCl}_{2}$ and a small incision was made to expose the dorsal surface of the circumesophageal nervous system, as shown in Figure 
$1 A$. Following the surgery, the animals were placed into a chamber containing $50 \mathrm{ml}$ of ASW. The exposed nervous system was visualized in infrared illumination provided by a 45 -W tungsten/halogen light source projected by a light guide through an infrared filter (Schott model $\mathrm{RG}-850$ ). A dissecting microscope formed an image of the nervous system in the infrared light upon a Dage MTi videocamera connected to a videomonitor and time-lapse videorecorder.

In vivo conditioning procedure. The details of the in vivo conditioning procedure have been described in a previous publication (Crow and Forrester, 1986) and will be described only briefly in this report. The protocols for conditioning and control procedures are shown in the diagram of Figure 2 . After exposure of the nervous system, the animals were placed in a chamber containing ASW and were dark adapted for 12 min (see Fig. 2). Immediately after the period of dark adaptation, the paired group received I trial of light (CS) paired with the application of $0.5 \mathrm{ml} \mathrm{5-HT}$. The 5-HT was applied to the region of the cerebropleural ganglion where 5-HT-immunoreactive varicosities and processes were identified in a previous immunocytochemistry study (Land and Crow, 1985). Serotonin was applied to the desired region of the nervous system by observing the image of the cerebropleural ganglion and injecting pipette on a videomonitor. The concentration of 5-HT was adjusted to yield a final concentration in the ASW bath of $0.1 \mathrm{~mm}$. Animals remained in the light in the presence of 5-HT for $5 \mathrm{~min}$, followed by an ASW rinse and an additional $12 \mathrm{~min}$ of dark adaptation. An unpaired control group received the 5 -min CS, followed by $5 \mathrm{~min}$ of dark adaptation before the application of 5-HT (see Fig. 2). The 5-HT was applied in the dark (infrared illumination) for the unpaired control group and remained in the ASW bath for $5 \mathrm{~min}$, followed by the application of normal ASW. Animals in the backward control group received 5-HT applied in the dark (infrared light), followed by normal ASW application and $5 \mathrm{~min}$ in the dark before the $5 \mathrm{~min} \mathrm{CS}$. The normal control group received only light without the application of 5-HT. The different treatment conditions were coded so that the subsequent cellular neurophysiological analysis was conducted without knowing whether an animal was from the paired, unpaired control, or backward control groups. The animals were not sutured after the treatment because the wound margins typically closed within several hours after surgery and appeared normal when examined $24 \mathrm{hr}$ after the treatment, as shown in the example in Figure $1 B$.

In order to assess the contribution of direct and indirect effects of 5-HT on type-B photoreceptors, the in vivo conditioning procedure was modified (shown in Fig. 8). After surgical exposure of the nervous system, 2 glass pipettes modified from patch-clamp electrodes were placed over each eye and proximal optic nerve. This procedure provided a tight seal between the pipette tip and base of the eye and optic nerve, as verified by injecting dye into the electrodes. The modification of the conditioning procedure consisted of pairing the CS with injection of 0.1 mM 5-HT into the electrodes containing the eyes. A control group received the 5 -min $\mathrm{CS}$, followed by $5 \mathrm{~min}$ of dark adaptation before injecting 5-HT into the electrode in the dark (infrared light).

Intracellular recording. Electrophysiological recordings were collected at 2 time periods following in vivo conditioning and control procedures. One hour or $24 \mathrm{hr}$ after the termination of the treatments, the circumesophageal nervous system was removed from the animals and pinned to a stage in a recording chamber filled with ASW. Experiments were conducted in buffered ASW (10 mM HEPES; $\mathrm{pH}, 7.6$ ) having the following composition (in $\mathrm{mM}$ ): $460 \mathrm{NaCl}, 10 \mathrm{KCl}, 10 \mathrm{CaCl}_{2}$, and $55 \mathrm{MgCl}_{2}$. The ASW solution was brought to $\mathrm{pH} 7.6$ at $15 \pm 0.5^{\circ} \mathrm{C}$ with dilute $\mathrm{NaOH}$. Identified type-B photoreceptors were isolated from synaptic interactions by cutting the optic nerve proximal to the cell body with a single-edge razor. The isolated nervous systems were incubated in a protease solution (Sigma Chemical Co; $0.67 \mathrm{mg} / \mathrm{ml}, 5-6 \mathrm{~min}$ ) to facilitate microelectrode penetration of identified B-photoreceptors. Glass microelectrodes used for intracellular recordings from isolated B-photoreceptors had resistances between 30 and $50 \mathrm{M} \Omega$ when filled with 4 $M$ potassium acetate. The temperature of the ASW in the recording chamber was maintained at $14-15^{\circ} \mathrm{C}$. Seawater temperature was monitored by a thermistor inserted into the recording chamber.

Light stimuli. Light steps $(2 \mathrm{~min})$ were delivered to the eyes by a tungsten/halogen source projecting through an optic fiber positioned above the preparation. The light was focused to a spot that covered the eye. The intensity of the light on the eye was $10 \times 10^{-4} \mathrm{~W} / \mathrm{cm}^{2}$ at 510 $\mathrm{nm}$, the wavelength used in all experiments. Photoreceptors were initially dark adapted for 15 min following electrode penetration before the delivery of the 2-min light step. After dark adaptation, light-evoked generator potentials were collected from identified isolated B-photoreceptors using standard intracellular recording procedures and microcomputer data acquisition and storage techniques. Short- and long-term enhancement is expressed in B-photoreceptors by increases in the amplitude of the peak and plateau phases of the generator potential. The transient peak represents the largest amplitude of the generator potential elicited at the onset of light measured from the dark-adapted resting potential. The plateau phase was determined by measuring the amplitude of generator potentials at the end of the 2-min light step. Both indices of enhancement are consistent with measurements of generator potential amplitude derived by integrating the voltage over time (Crow et al., 1991).

Four of the 5 photoreceptors within each eye of Hermissenda can be visually identified based upon their positions in the lateral, medial, anterior, and posterior part of the eye. For descriptive purposes, type-B photoreceptors located in the lateral-posterior and medial-posterior position in each eye will be referred to as lateral B-photoreceptors and medial B-photoreceptors, respectively. Different identified photoreceptors were examined following in vivo conditioning because it is not known if different B-photoreceptors within each eye represent a distinct and unique functional cell type or if they exhibit different potentials for the expression of short- and long-term enhancement.

Statistical analysis. A one-way analysis of variance was used to examine overall differences in the peak and plateau phases of generator potentials recorded from preparations that received paired, unpaired control, backward control, and normal control conditions. Data were analyzed separately for generator potentials collected at $1 \mathrm{hr}$ (shortterm) and $24 \mathrm{hr}$ (long-term) after 1-trial conditioning. Following significant overall effects, paired comparisons involved the application of Dunnett's $t$ statistic (Winer, 1962).

\section{Results}

The results of the analysis of neural correlates recorded from medial and lateral B-photoreceptors following in vivo conditioning are presented in 2 parts. In the first part, characteristics of light-evoked generator potentials recorded from isolated medial and lateral B-photoreceptors are described for paired, unpaired control, backward control, and normal control groups 1 hr after the conclusion of the different treatments. Increases in the amplitude of generator potentials detected $1 \mathrm{hr}$ but not 24 $\mathrm{hr}$ after in vivo conditioning are referred to as short-term enhancement. In the second phase of the analysis, generator potentials recorded $24 \mathrm{hr}$ following in vivo conditioning and control procedures are described. Increases in the amplitude of generator potentials recorded from identified photoreceptors $24 \mathrm{hr}$ after conditioning are described as long-term enhancement.

Short-term enhancement. The statistical analysis of the amplitude of generator potentials evoked by light $1 \mathrm{hr}$ after the conclusion of in vivo conditioning revealed that light responses were enhanced for both medial and lateral B-photoreceptors. Examples of light-evoked generator potentials from 4 different medial B-photoreceptors recorded after the paired, unpaired control, backward control, and normal control procedures are shown in Figure 3. The generator potentials recorded from the paired, unpaired control, and backward control groups were enhanced as compared to the example from a normal control group (Fig. $3 D$ ). The mean amplitude of the peak and plateau phases of light-evoked generator potentials for the paircd, unpaired control, backward control, and normal control groups is shown in Table 1. Generator potential peak amplitudes recorded from groups that received light paired with 5-HT $(N=17)$, unpaired light and 5-HT $(N=19)$, the backward control procedure $(N=6)$, and normal controls $(N=12)$ showed significant overall effects $\left(F_{3,50}=5.85 ; p<0.01\right)$. Comparing the different treatments to the normal controls revealed that the paired group $(t=4.33 ; p<0.005)$, unpaired control group $(t=2.72 ; p<$ 

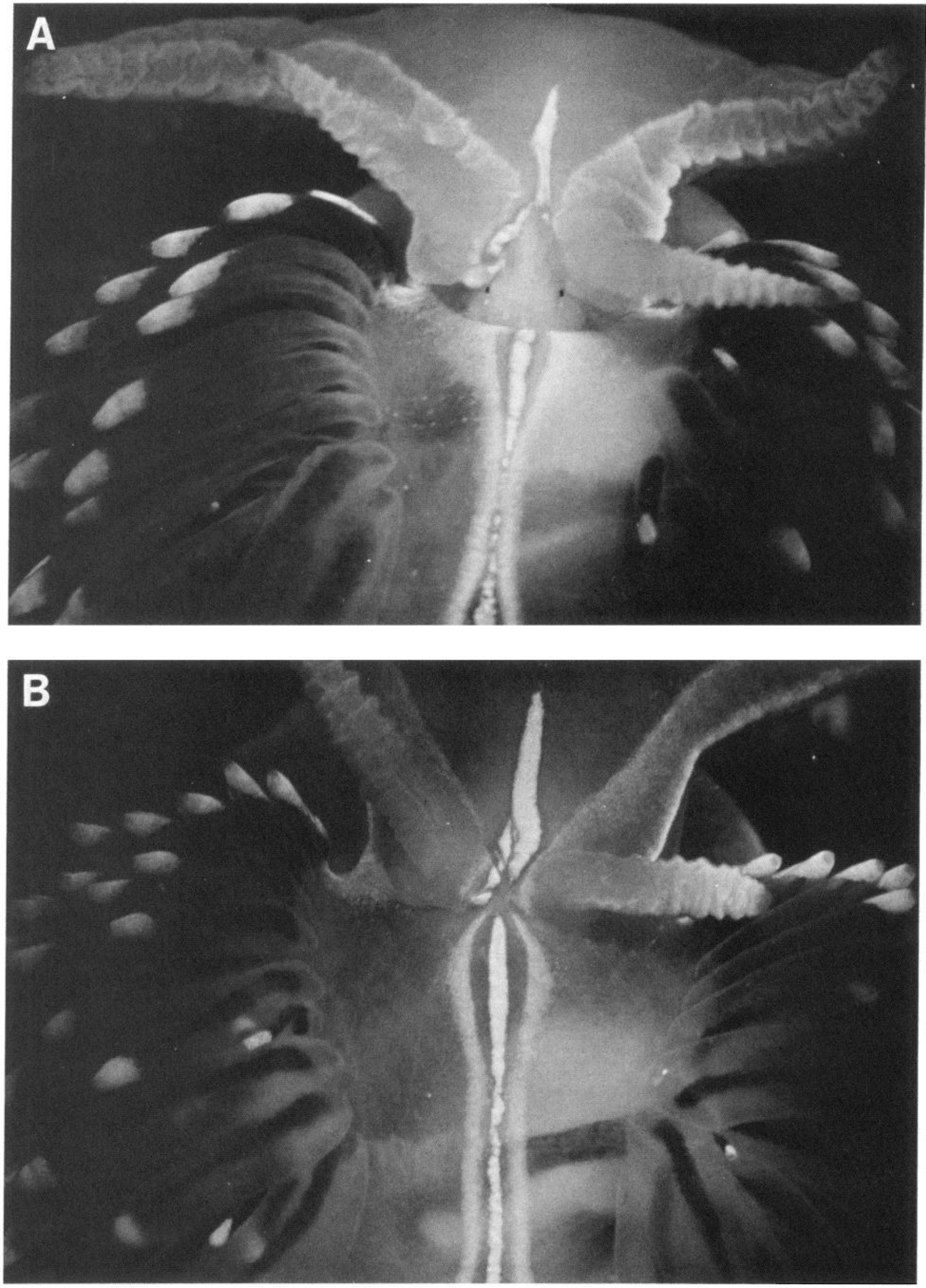

Figure 1. Photograph of the experimental preparation used to study short- and long-term enhancement following 1-trial in vivo conditioning. A, An example of a Hermissenda immediately following completion of a dorsal-lateral incision to expose the circumesophageal nervous system. The 


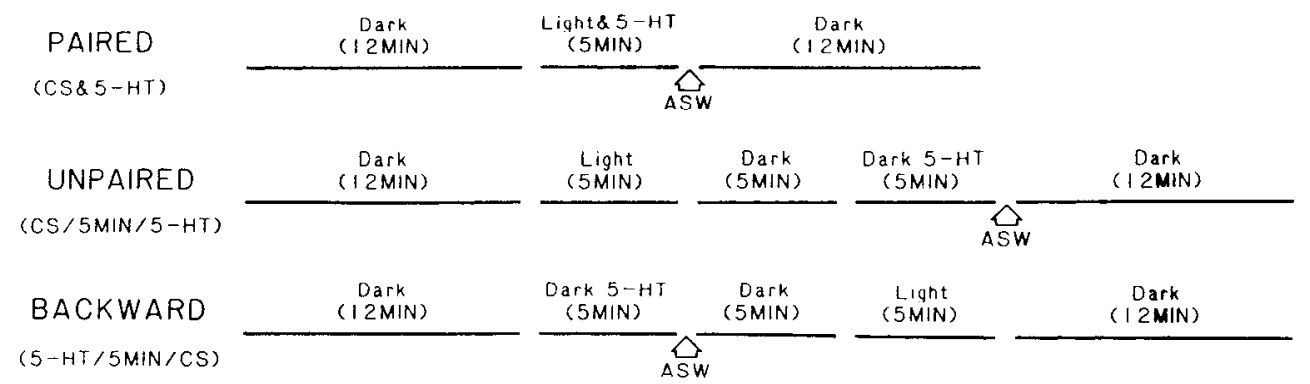

Figure 2. Experimental protocol for investigating 1-trial in vivo conditioning. Animals were assigned to 1 of 3 different treatment conditions. Following 12 min of dark adaptation, the paired light (CS) and 5-HT group received 15 -min trial of light paired simultaneously with the injection of 5-HT onto the exposed nervous system, followed by a wash with normal ASW and 12 min in the dark. The unpaired control group received the light $\mathrm{CS}(5 \mathrm{~min})$ followed by a 5 -min period in the dark before applying 5 -HT ( $5 \mathrm{~min}$ ) to the nervous system in the dark (infrared illumination). The backward control group received the 5 -HT $(5 \mathrm{~min})$ in the dark followed by a 5 -min period of dark adaptation in normal ASW before presenting the light (CS).

$0.025)$, and backward control group $(t=2.59 ; p<0.05)$ were significantly different from normal controls.

The analysis of the plateau phase of the generator potential revealed that the normal controls were significantly different from the paired group $(t=2.48 ; p<0.05)$, the unpaired control group $(t=2.46 ; p<0.05)$, and the backward control group ( $t$ $=2.45 ; p<0.05$ ). However, the peak and plateau phases of generator potentials recorded from medial B-photoreceptors from the paired, unpaired control, and backward control groups were not significantly different from each other. These results indicate that short-term enhancement detected in medial B-photoreceptors is not dependent upon pairing light and 5-HT, and enhancement is expressed by an increase in both the peak and plateau phases of light-evoked generator potentials. We next examined generator potentials recorded from lateral B-photoreceptors cvoked by light $1 \mathrm{hr}$ following the application of light and 5-HT. Generator potentials recorded from lateral B-photoreceptors from the paired, unpaired, and backward control groups were enhanced as compared to normal controls. The mean amplitude of the peak and plateau phase of the generator potentials recorded from lateral B-photoreceptors is shown in Table 1. The statistical analysis revealed an overall difference between the paired $(N=23)$, unpaired control $(N=22)$, backward control $(N=10)$, and normal control groups $\left(N=12 ; F_{3.63}\right.$ $=2.98 ; p<0.05)$. Comparisons between the various treatment groups and normal controls showed that the plateau phases from paired $(t=2.93 ; p<0.01)$, unpaired control $(t=2.4 ; p<0.05)$, and backward control groups $(t=2.32 ; p<0.05)$ were significantly different from normal controls, while none of the treatments differed from each other.

Consistent with the analysis of the plateau phase was the finding that the peak amplitude of the generator potential rccorded from the paired $(t=3.07 ; p<0.005)$, unpaired control $(t=2.28 ; p<0.05)$, and backward control groups $(t=2.1 ; p$ $<0.05)$ were significantly larger as compared to normal controls. These results indicate that the presentation of light and 5-HT produces a non-pairing-specific enhancement of the peak and plateau phase of generator potentials in both lateral and medial type-B photoreceptors.
Long-term enhancement. Our previous research has shown that pairing light with the direct application of 5-HT to the exposed nervous system produced a pairing-specific change in phototactic behavior detected $24 \mathrm{hr}$ after in vivo conditioning. To examine possible long-term neural correlates of in vivo conditioning, we recorded generator potentials from identified and isolated medial and lateral B-photoreceptors following treatment and control procedures.

Examples of light-evoked generator potentials recorded from medial B-photoreceptors of paired and unpaired control groups collected $1 \mathrm{hr}$ and $24 \mathrm{hr}$ following the conclusion of in vivo conditioning are shown in Figures 4 and 5. In contrast to the results of the analysis of generator potential amplitudes at $1 \mathrm{hr}$ after conditioning, we found that in vivo conditioning does not produce long-term enhancement of generator potentials from medial B-photoreceptors recorded $24 \mathrm{hr}$ after conditioning. This is supported by the statistical analysis, which did not reveal overall significant differences between the treatment and control groups. Group means for the peak and plateau phases of the generator potential recorded from medial B-photoreceptors are shown in Table 2. Paired comparisons showed that the paired $(N=11)$ and unpaired control groups $(N=11)$ were not significantly different from each other or normal controls $(N=12)$. These results show that in vivo conditioning produces only a short-term enhancement of generator potentials recorded from medial B-photoreceptors. The comparison between the peak amplitude of the generator potential recorded $1 \mathrm{hr}$ and $24 \mathrm{hr}$ after conditioning provides additional support for this conclusion. The peak amplitude of the generator potential was significantly smaller for the paired group $24 \mathrm{hr}$ after conditioning as compared to response recorded $1 \mathrm{hr}$ after conditioning $(t=2.69$; $p<0.025)$. Similar results were obtained from the analysis of the plateau phase of the generator potential $(t=2.36 ; p<0.05)$. The paired and unpaired control groups were not significantly different from each other or normal controls $24 \mathrm{hr}$ after in vivo conditioning.

Examples of generator potentials recorded from 2 different lateral B-photoreceptors $24 \mathrm{hr}$ after paired and unpaired light and 5-HT are shown in Figure 6. In contrast to the results of

in vivo conditioning procedure consists of pairing light with the direct application of 5-HT to the exposed nervous system of otherwise intact animals. $B$. A photograph of the preparation as shown in $A$ taken $24 \mathrm{hr}$ after exposure of the nervous system, showing that the wound margins have closed and the animal appears normal. Intracellular recordings are collected from identified photoreceptors within the eyes of preparations that have recovered as indicated in $B$. 
Figure 3. Short-term enhancement of light-evoked generator potentials recorded from surgically isolated medial B-photoreceptors $1 \mathrm{hr}$ after in vivo conditioning. $A$, Intracellular recording of a generator potential from an isolated medial B-photoreceptor evoked by a 2-min light step $1 \mathrm{hr}$ following the presentation of light paired with 5-HT. $B$, Intracellular recording of a light-evoked generator potential from a medial B-photoreceptor $1 \mathrm{hr}$ after unpaired light and 5-HT. $C$, Generator potential evoked by a 2 -min light step recorded from a medial B-photoreceptor $1 \mathrm{hr}$ following the backward control procedure. $D$. Generator potential of a medial B-photoreceptor recorded from a normal control preparation. The amplitudes of the generator potentials shown in the examples in $A-C$ are enhanced as compared to the normal control shown in $D$. The short dashed lines in the recordings in $A-C$ represent the peak and plateau amplitude of the normal control shown in $D$.
A

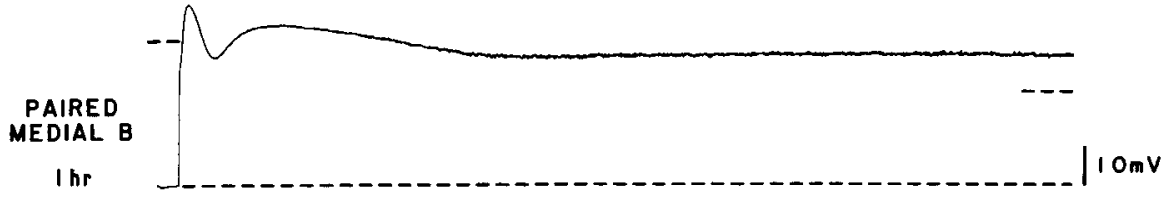

B

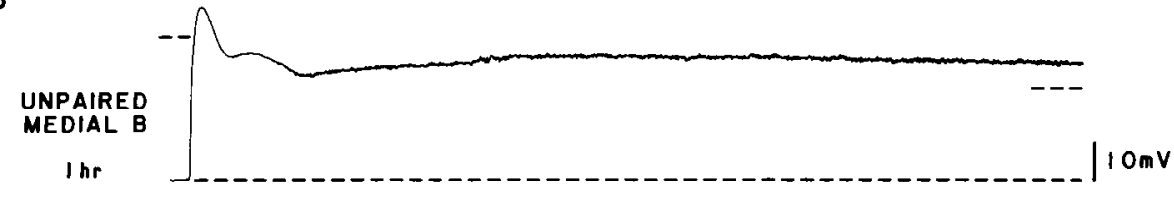

C

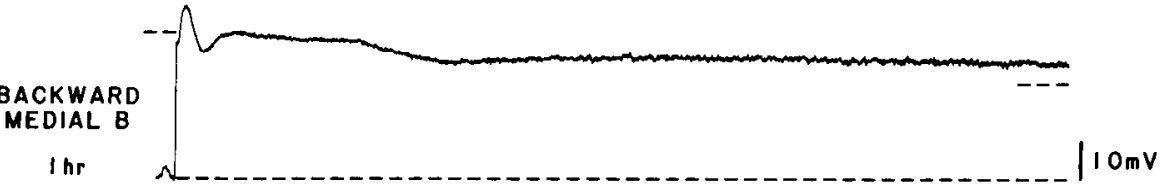

D

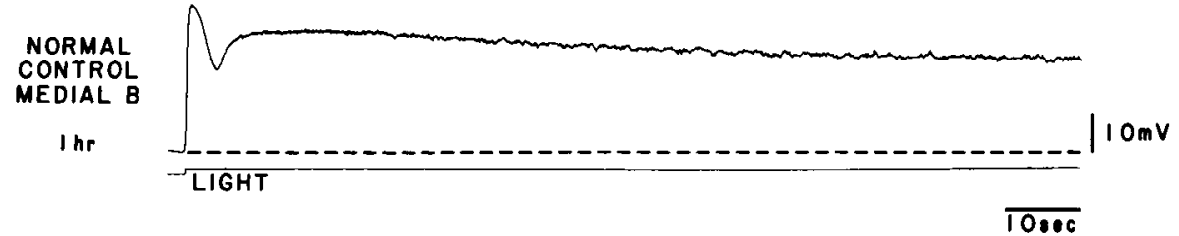

the analysis of light-evoked generator potentials from medial B-photoreceptors, lateral B-photoreceptors exhibited a long-term enhancement. In addition, unlike short-term enhancement, longterm enhancement detected in lateral B-photoreceptors is specific to pairing light and 5-HT (Fig. 7). The statistical analysis of the generator potential peak revealed that there were no overall significant differences. The paired $(N=26)$, unpaired control $(N=23)$, and backward control groups $(N=5)$ were not significantly different from each other or normal controls $(N-12)$. However, the analysis of the plateau phase measured at the end of the 2-min light step revealed an overall significant difference $\left(F_{3,62}=5.93 ; p<0.01\right)$. Paired comparisons showed that the plateau phase of the paired group was significantly larger than the normal control $(t=3.66 ; p<0.005)$, unpaired control $(t=$ $3.32 ; p<0.005)$, and backward control group $(t=2.82 ; p<$ 0.01 , while the unpaired and backward controls were not significantly different from normal controls. These results show that long-term enhancement is expressed only in lateral B-photoreceptors by a larger amplitude of the plateau phase of the generator potential (Fig. 7).

In summary, the results indicate that in vivo conditioning produces 2 effects in identified photoreceptors within the eyes of Hermissenda. One effect is a short-term enhancement of generator potentials detected in both lateral and medial B-photoreceptors. Short-term enhancement does not depend upon pairing light with 5-HT because paired, unpaired control, and

Table 1. Short-term enhancement of generator potentials

\begin{tabular}{|c|c|c|c|c|}
\hline \multirow[b]{2}{*}{ Experimental condition } & \multicolumn{2}{|c|}{ Medial type-B photoreceptors } & \multicolumn{2}{|c|}{ Lateral type-B photoreceptors } \\
\hline & $\overline{\text { Peak }}$ & Plateau & Peak & Plateau \\
\hline Paired & $\begin{array}{l}44.3 \pm 1.20^{*} \\
(n=17)\end{array}$ & $29.9 \pm 0.66^{*}$ & $\begin{array}{l}43.3 \pm 0.71^{*} \\
(n=23)\end{array}$ & $29.3 \pm 0.72^{*}$ \\
\hline Unpaired control & $\begin{array}{l}43.1 \pm 0.84^{*} \\
(n=19)\end{array}$ & $28.5 \pm 0.49^{*}$ & $\begin{array}{l}42.9 \pm 0.78^{*} \\
(n=22)\end{array}$ & $28.9 \pm 1.08^{*}$ \\
\hline Backward control & $\begin{array}{l}42.6 \pm 1.09^{*} \\
(n=6)\end{array}$ & $28.5 \pm 0.51^{*}$ & $\begin{array}{l}42.5 \pm 1.2^{*} \\
(n=10)\end{array}$ & $28.7 \pm 0.93^{*}$ \\
\hline Normal control & $\begin{array}{l}38.2 \pm 0.35 \\
(n=12)\end{array}$ & $25.5 \pm 1.03$ & $\begin{array}{l}39.0 \pm 1.1 \\
(n=12)\end{array}$ & $24.7 \pm 0.99$ \\
\hline
\end{tabular}

This table shows the mean amplitude ( $\mathrm{mV} \pm \mathrm{SEM}$ ) of the generator potential peak and plateau phase evoked by a 2 -min light step recorded from medial and lateral B-photoreceptors $1 \mathrm{hr}$ after the experimental and control procedures described in Materials and Methods.

* Indicates comparisons that are significantly different from normal controls as described in Results $(n=$ number of photoreceptors). 
A

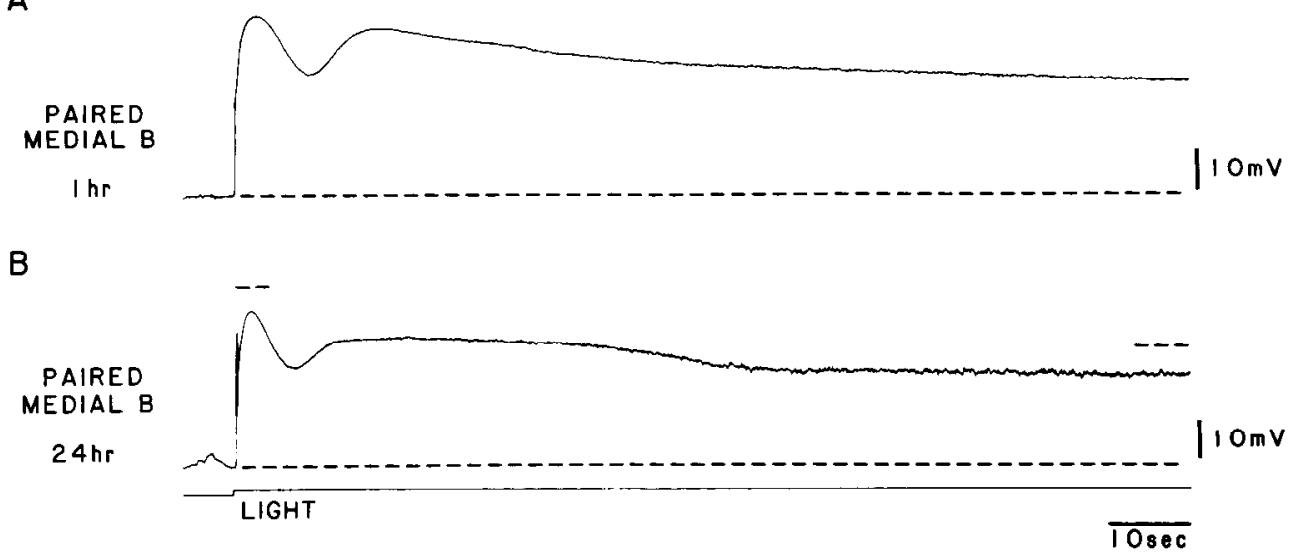

Figure 4. Medial B-photoreceptors express short-term enhancement, but not long-term enhancement, $24 \mathrm{hr}$ following in vivo conditioning. $A$, Generator potential from a medial B-photoreceptor evoked by the light step I hr following the presentation of light paired with 5-HT. $B$, Generator potential recordcd from a medial B-photorcceptor $24 \mathrm{hr}$ following paired light and 5-HT. The short dashed lines in the recording in $B$ represent the peak and plateau amplitude of the generator potential shown in $A$. The generator potential shown in $A$ is enhanced as compared to the example shown in $B$. These results show that medial B-photoreceptors do not exhibit long-term enhancement. backward control results were similar. The second effect, which was observed only in lateral B-photoreceptors, is a long-term enhancement of the generator potential that depends upon pairing light with 5-HT. The long-term enhancement is expressed by an increase in the amplitude of the steady-state plateau phase of the generator potential.

Assessment of direct effects. Long-term enhancement may be the result of either a direct effect of 5-HT upon lateral B-photoreceptors or indirect effects mediated by neurons presynaptic to the photoreceptors that are activated by $5-\mathrm{HT}$. In order to examine this issue, we paired light with the direct application of 5-HT to the eyes using the procedures shown in the diagram in Figure 8. Significant long-term enhancement of the plateau phase of the generator potential was observed for the group that reccived the CS paired with injection of 5-HT into the pipettes $(N=6)$, as compared to an unpaired control group $\left(N=7 ; t_{11}\right.$ $=3.04 ; p<0.01$ ). Group means of the plateau phase for the paired and unpaired control groups are shown in Figure 9. The enhanced plateau phase of the generator potential from the paired group as compared to the mean amplitude of the plateau phase for the unpaired control group indicates that direct application of 5-HT to lateral B-photoreceptors when paired with the CS is sufficient to produce a significant long-term enhancement of generator potentials.

\section{Discussion}

One trial in vivo conditioning: relationship to behavior

We have found that in vivo conditioning produces both shortand long-term enhancement of generator potentials in different identified photoreceptors within the visual system of Hermissenda. Because the enhanced generator potentials are expressed after conditioning in isolated B-photoreceptors, the changes are intrinsic and, once induced, are not due to an alteration in synaptic input from neurons that are presynaptic to the photoreceptors. This finding suggests that long-term enhancement may contribute to the modification of phototactic behavior produced by in vivo conditioning because postsynaptic neurons that contribute to the locomotor circuit would be expected to receive enhanced synaptic input from photoreceptors. However, the precise postsynaptic action of enhancement in the visual system has not been determined. In an earlier report, we presented evidence that the exogenous application of 5-HT, when paired with the CS, can effectively serve as an unconditioned stimulus (US) to produce long-term phototactic suppression (Crow and
Forrester, 1986). Because the component of phototactic behavior that is modified by in vivo conditioning has been shown previously to exhibit long-term phototactic suppression following $3 \mathrm{~d}$ of behavioral conditioning, it is attractive to suggest that a common mechanism may underlie both examples of conditioning. In fact, it has been suggested that 5-HT may be the transmitter in the US pathway. Consistent with this view are recent reports of the involvement of 5-HT in in vitro conditioning of the isolated nervous system (Grover et al., 1989) and evidence for serotonergic modulation of membrane conductances of B-photoreceptors (Crow and Bridge, 1985; Farley and Wu, 1989; Acosta-Urquidi and Crow, 1990). Additional support for this hypothesis comes from cellular studies, where there is now a considerable amount of evidence indicating that classical conditioning produces cellular correlates that are intrinsic to the type-B photoreceptors in Hermissenda (for a recent review, see Crow, 1988). While it is attractive to suggest that 5-HT plays a role in classical conditioning of Hermissenda, there are reported differences in the cellular correlates produced by 1-trial in vivo conditioning and the conditioning procedure consisting

Table 2. Generator potential amplitude $24 \mathrm{hr}$ following in vivo conditioning

\begin{tabular}{|c|c|c|c|}
\hline \multirow{2}{*}{$\begin{array}{l}\text { Experimental } \\
\text { condition }\end{array}$} & \multicolumn{2}{|c|}{ Medial type-B photoreceptors } & \multirow{2}{*}{$\begin{array}{l}\begin{array}{l}\text { Lateral } \\
\text { type-B } \\
\text { photorcceptors }\end{array} \\
\text { Peak }\end{array}$} \\
\hline & Peak & Plateau & \\
\hline Paired & $\begin{array}{l}40.7 \pm 1.7 \\
(n=11)\end{array}$ & $25.1 \pm 2.06$ & $\begin{array}{l}40.1 \pm 1.01 \\
(n=26)\end{array}$ \\
\hline Unpaired control & $\begin{array}{l}40.5 \pm 0.91 \\
(n=11)\end{array}$ & $26.0 \pm 2.2$ & $\begin{array}{l}39.9 \pm 0.58 \\
(n=23)\end{array}$ \\
\hline Backward control & $\begin{array}{l}39.5 \pm 2.0 \\
(n=2)\end{array}$ & $25.6 \pm 1.8$ & $\begin{array}{l}40.7 \pm 2.4 \\
(n=5)\end{array}$ \\
\hline Normal control & $\begin{array}{l}39.2 \pm 0.53 \\
(n=12)\end{array}$ & $25.9 \pm 1.2$ & $\begin{array}{l}40.2 \pm 1.8 \\
(n=12)\end{array}$ \\
\hline
\end{tabular}

This table shows the mean amplitude ( $\mathrm{mV} \pm \mathrm{SEM}$ ) of generator potential peak and plateau phase evoked by a 2 -min light step recorded from medial B-photoreceptors and peak phase recorded from lateral B-photoreceptors $24 \mathrm{hr}$ after the experimental and control procedures described in Materials and Methods. The mean amplitude of the plateau phase for lateral B-photoreceptors is shown in Figure 7. None of the comparisons were significantly different from normal controls as described in Results ( $n=$ number of photoreceptors). 
A

Figure 5. Medial B-photoreceptors exhihit short-term enhancement, but not long-term enhancement, $24 \mathrm{hr}$ after unpaired light and 5-HT. $A$, Generator potential from a medial $B$-photoreceptor evoked by the light step $1 \mathrm{hr}$ after the presentation of unpaired light and 5-HT. $B$, Generator potential recorded from a medial B-photoreceptor $24 \mathrm{hr}$ after unpaired light and 5-HT. The short dashed lines in the recording in $B$ represent the peak and plateau amplitude of the generator potential shown in $A$ The generator potential is enhanced at $1 \mathrm{hr}$ but not at $24 \mathrm{hr}$.

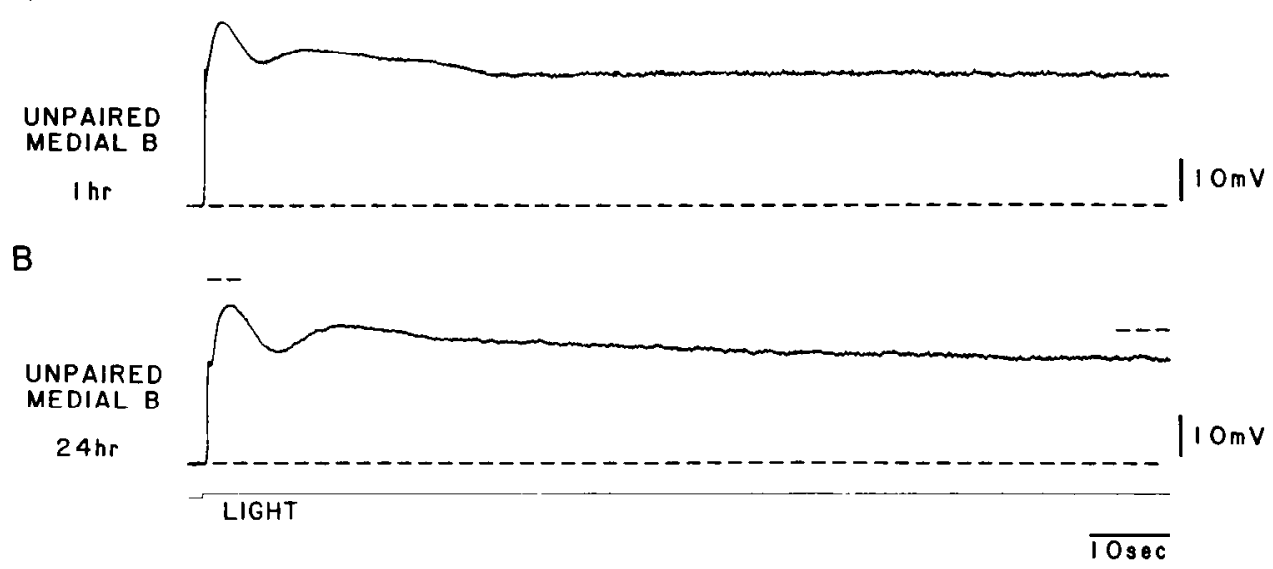

of multiple-session training. Multiple-session training consists of 50 trials of the CS paired with the US (high-speed rotation) each day, for 3 consecutive days (Crow and Alkon, 1978). First, it has been reported that 150 trials of multiple-session training produces a reduction in the amplitude of the steady-state plateau phase of light-evoked generator potentials measured after $5 \mathrm{~min}$ of illumination (Crow, 1985). We suggested that long-term phototactic suppression produced by conditioning may be explained by the CS eliciting a smaller generator potential (Crow, 1985). Second, it was rccently reported that only medial B-photoreceptors exhibit enhanced generator potentials and a concomitant reduction in $2 \mathrm{~K}^{+}$currents detected $24 \mathrm{hr}$ after 150 trials of multiple-session training (Alkon et al., 1985). These findings suggested that phototactic suppression was due to enhanced B-photoreceptor inhibition of type-A photoreceptor output. Thus, light-dependent excitation of medial type-A photoreceptors would be diminished by enhanced type-B inhibition of medial A-photoreceptors that project via interneurons to motor neurons (Alkon, 1984; Alkon et al., 1985). However, neural correlates of conditioning detected in putative motor neurons are inconsistent with this interpretation. It was reported that, after classical conditioning, the activity recorded from identified pedal neurons elicited by the CS (light) was reduced below the frequency of spontaneous activity recorded in the dark (Crow, 1981). Because type-A photoreceptors do not typically discharge action potentials spontaneously in the dark, their activity during illumination cannot be less than their activity in the dark. In addition, previous research has shown that behavior in response to the CS is suppressed by conditioning, while locomotion in the dark is not significantly changed after conditioning (Crow and Offenbach, 1983). These results suggest that B-photoreceptors may project to interneurons that inhibit pedal motor neurons or project to spontaneously active interneurons that are inhibited by type-B photoreceptor synaptic input. Moreover, different identified type-B photoreceptors may project to different postsynaptic neurons with different motor projections. Thus, differences in cellular correlates produced by the 2 procedures may indicate that a complex behavioral response such as visually guided locomotion can be modified by different cellular mechanisms contributing to the same behavioral outcome. Taken collectively, the present findings may indicate that 1-trial in vivo conditioning produces phototactic suppression by a mechanism quite different from the one produced by multiplesession conditioning. However, because little is known about cellular correlates produced by a single conditioning session, it may be premature to conclude that the mechanisms are, in fact, different. While there are clear differences in outcomes, there are some interesting similarities between single-session training and 1-trial in vivo conditioning (see next section). A satisfactory explanation for phototactic suppression requires an understand-
Figure 6. Long-term enhancement of light-evoked generator potentials recorded from surgically isolated lateral B-photoreceptors $24 \mathrm{hr}$ following in vivo conditioning. $A$, Generator potential from an isolated lateral B-photoreceptor evoked by light $24 \mathrm{hr}$ following the presentation of light paired with 5-HT. $B$, Generator potential recorded from a lateral B-photoreceptor $24 \mathrm{hr}$ following unpaired light and 5-HT. The generator potential in $A$ exhibits an enhanced plateau as compared to the example shown in $B$. The peak of the generator potential recorded from lateral B-photoreceptors does not show long-term enhancement. The short dashed line in $B$ represents the amplitude of the generator potential plateau shown in $A$.
A

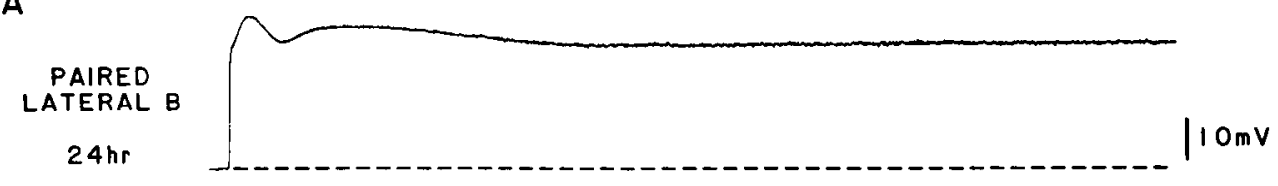

B

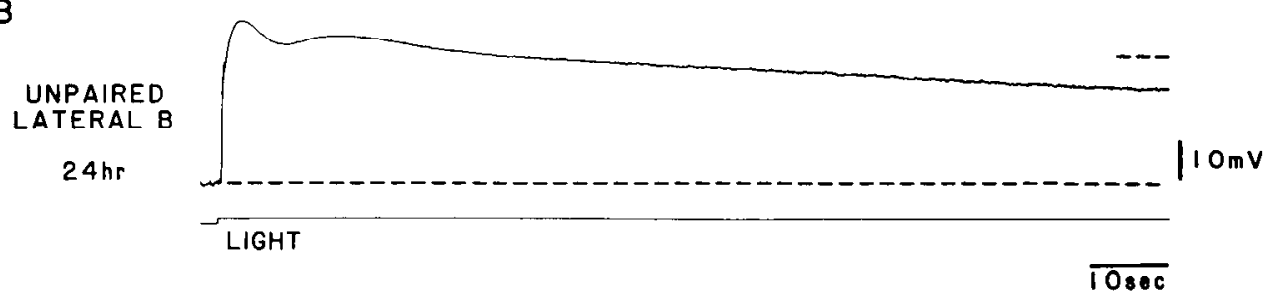




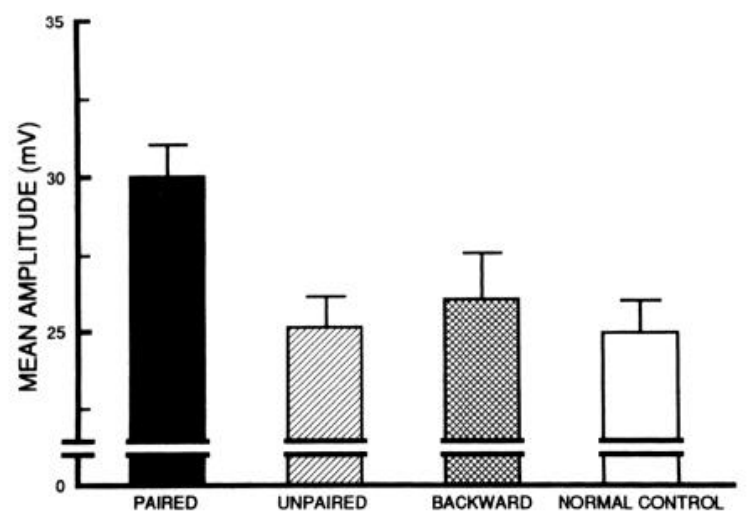

Figure 7. Group data showing the mean amplitude $(\mathrm{mV} \pm \mathrm{SEM})$ of the generator potential plateau phase recorded from lateral B-photoreceptors for the groups that received the CS paired with 5-HT $(N=$ 26), the unpaired CS and 5-HT control $(N=23)$, the backward control group $(N=5)$, and normal control treatment $(N=12)$. The plateau phase of the generator potential is significantly enhanced for the paired light and 5-HT group as compared to the different control groups.

ing of how the activity of photoreceptors within the visual system can influence the activity of the neural network responsible for locomotion in Hermissenda. To date, the interactions between photoreceptors and postsynaptic neurons in the circuit generating locomotion are not known; however, such studies have been initiated (Crow, 1981; Goh and Alkon, 1984; Hodgson and Crow, 1987; Richards and Farley, 1987).

\section{One-trial in vivo conditioning and single-session training}

It is of interest to note some of the similarities between 1-trial in vivo conditioning and studies of single-session conditioning in Hermissenda. First, we reported that 5 and 10 conditioning trials resulted in significant short-term nonassociative changes in phototactic behavior (Crow, 1983). These results indicated that the nonassociative contribution to phototactic behavior was expressed soon after training and decremented rapidly following the conclusion of both single-session and multiple-session training. Our present results show that immediate or short-term enhancement is not dependent upon pairing (nonassociative), and that the pairing-dependent enhancement is expressed at 24 $\mathrm{hr}$, a time when the short-term nonassociative enhancement has decremented. These studies suggested that, because associative and nonassociative components of phototactic suppression follow different time courses, the underlying mechanisms may be independent; that is, the mechanism for the associative effect may not be an elaboration of the nonassociative mechanism. It is interesting to note that the nonassociative correlate of in vivo conditioning is expressed before the long-term associative correlate. However, we have evidence that long-term enhancement is independent of short-term nonassociative enhancement (see next section).

\section{Short- and long-term enhancement: possible mechanisms}

The suggestion that short- and long-term enhancement may involve different mechanisms is supported by our recent cellular studies. First, we have shown that long-term enhancement depends upon protein synthesis, while short-term enhancement does not (Crow and Forrester, 1990). Second, we have examined the role of activation of protein kinases in the induction and expression of short- and long-term enhancement (Crow and

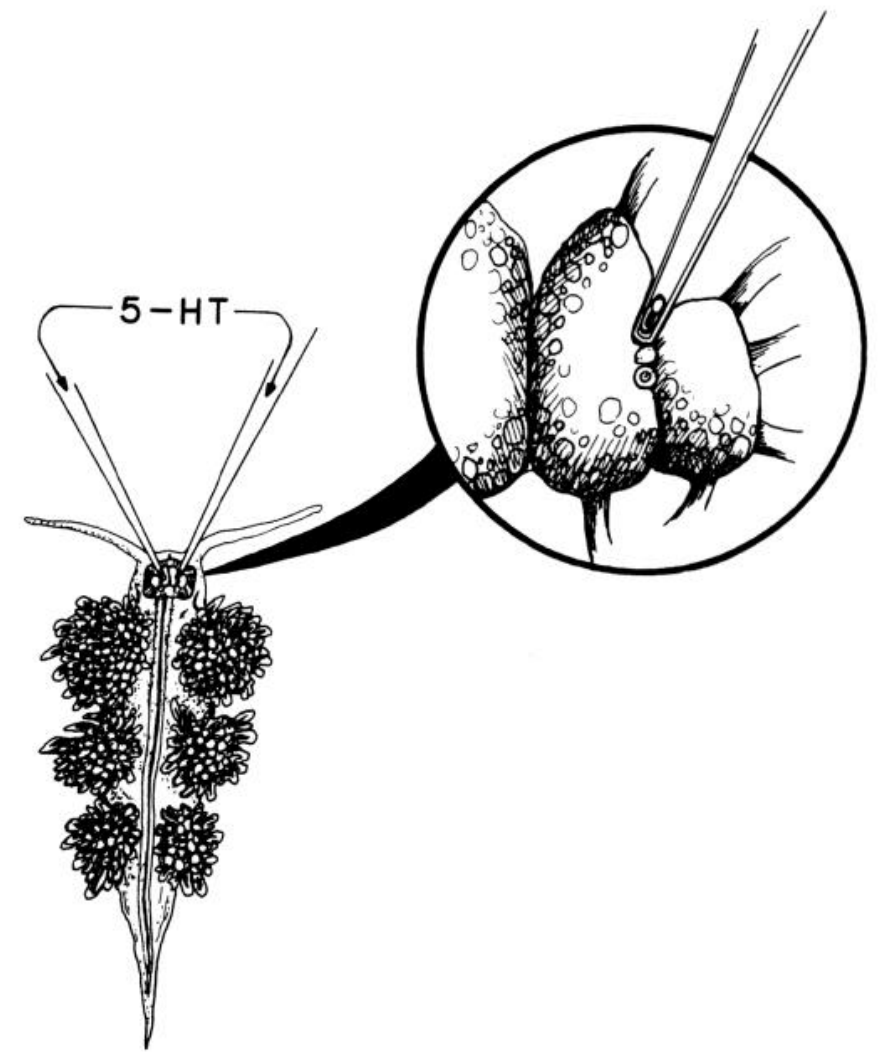

Figure 8. Drawing of modification of the in vivo conditioning procedure designed to restrict stimulation with light and 5-HT to the visual system. Two glass pipettes, prepared from patch-clamp electrodes and mounted in micromanipulators, were placed over each eye of a surgically prepared animal. Illumination of the eyes was paired with the injection of $0.1 \mathrm{~mm} 5$-HT into each pipette. An unpaired control group received $5 \mathrm{~min}$ of light and a 5-min period of dark adaptation before the 5-HT injection in the dark (infrared illumination; see protocol in Fig. 2).

Forrester, 1988; Forrester and Crow, 1988, 1989; Crow et al., 1991). Numerous studies have implicated the $\mathrm{Ca}^{2+} /$ phospholipid-dependent kinase protein kinase $\mathrm{C}$ (PKC) in cellular regulation and synaptic and cellular plasticity (Berridge, 1986; Farley

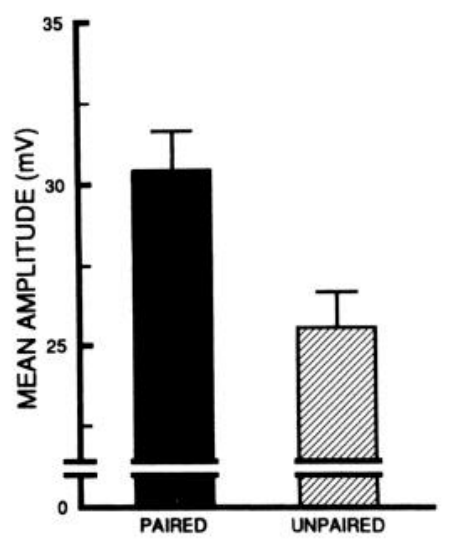

Figure 9. Group data showing the mean amplitude $(\mathrm{mV} \pm \mathrm{SEM})$ of the generator potential plateau phase recorded from lateral B-photoreceptors for the groups that received the CS paired with 5-HT $(N=6)$ and the unpaired CS and 5-HT $(N=7)$. The paired group showed significant enhancement $(p<0.01)$ as compared to the unpaired control group. The direct application of 5-HT to the lateral B-photoreceptors when paired with the CS is sufficient to produce long-term enhancement. 
and Auerbach, 1986; Neary et al., 1986; Alkon et al., 1988; Crow, 1988; Nishizuka, 1988; Bank et al., 1989). We found that short- and long-term enhancement can be induced by pairing the CS with activation of PKC with diacylglycerol analogs or phorbol esters (Crow and Forrester, 1988). Furthermore, the induction of short-term enhancement is dependent upon activation of $\mathrm{PKC}$ because kinase inhibitors and downregulation of PKC block enhancement (Forrester and Crow, 1988; Crow et al., 1991). Interestingly, the conditions that are sufficient to block the induction of short-term enhancement do not block long-term enhancement (Forrester and Crow, 1988). Taken collectively, the results described above and our present results showing that short- and long-term enhancement are expressed in different identified B-photoreceptors suggest that long-term enhancement may depend upon a parallel signaling system that is independent of the processes responsible for the induction of short-term enhancement. These results have implications for models of short- and long-term memory, suggesting that the mechanisms for long-term memory may be independent from short-term memory.

\section{References}

Acosta-Urquidi J, Crow T (1990) 5-HT modulates two distinct $\mathrm{K}^{+}$ currents in Hermissenda type B photoreceptors. Soc Neurosci Abstr $16: 20$.

Acosta-Urquidi J, Sahley CL, Kleinhaus A (1989) Serotonin differentially modulates two potassium currents in an identified neuron in the leech. J Exp Biol 145:403-417.

Adolph AR, Tuan FJ (1972) Serotonin and inhibition in Limulus lateral eye. J Gen Physiol 60:679-697.

Alkon DL (1984) Calcium-mediated reduction of ionic currents: a biophysical memory trace. Science 226:1037-1045.

Alkon DL, Sakakibara M, Forman R, Harrigan J, Lederhendler I, Farley $J$ (1985) Reduction of two voltage-dependent $\mathrm{K}^{+}$currents mediates retention of a learned association. Behav Neurol Biol 44:278-300.

Alkon DL, Naito S, Kubota M, Chen C, Bank B, Smallwood J, Gallant $\mathrm{P}$, Rasmussen $\mathrm{H}$ (1988) Regulation of Hermissenda $\mathrm{K}^{+}$channels by cytoplasmic and membrane-associated C-kinase. J Neurochem 51: 903-917.

Bank B, LoTurco JJ, Alkon DL (1989) Learning-induced activation of protein kinase C: a molecular memory trace. Mol Neurobiol 3:5570.

Barlow RB Jr, Chamberlain SC, Kaplan E (1977) Efferent inputs and serotonin enhance the sensitivity of the Limulus lateral eye. Biol Bull $153: 414$.

Berridge M (1986) Second-messenger dualism in neuromodulation and memory. Nature 323:294-295.

Corrent G, McAdoo DJ, Eskin A (1978) Serotonin shifts the phase of the circadian rhythm from the Aplysia eye. Science 202:977-979.

Crow $T$ (1981) Neurophysiological correlates of conditioning in identified putative motorneurons in Hermissenda. Soc Neurosci Abstr 7: 352.

Crow T (1983) Conditioned modification of locomotion in Hermissenda crassicornis: analysis of time-dependent associative and nonassociative components. J Neurosci 12:2621-2628.

Crow T (1985) Conditioned modification of phototactic behavior in Hermissenda. II. Differential adaptation of B-photoreceptors. J Neurosci 5:215-223.

Crow T (1988) Cellular and molecular analysis of associative learning and memory in Hermissenda. Trends Neurosci 11:136-142.

Crow T, Alkon DL (1978) Retention of an associative behavioral change in Hermissenda. Science 201:1239-1241.

Crow T, Bridge MS (1985) Serotonin modulates photoresponses in Hermissenda type-B photoreceptors. Neurosci Lett 60:83-88.

Crow T, Forrester J (1986) Light paired with serotonin mimics the effect of conditioning on phototactic behavior of Hermissenda. Proc Natl Acad Sci USA 83:7975-7978.

Crow T, Forrester J (1988) Light paired with activators of protein kinase $\mathrm{C}$ produce short- and long-term enhancement of light responses of lateral B-photoreceptors in Hermissenda. Soc Neurosci Abstr 14: 839.

Crow T, Forrester J (1990) Inhibition of protein synthesis blocks longterm enhancement of generator potentials produced by one-trial in vivo conditioning in Hermissenda. Proc Natl Acad Sci USA 87:44904494

Crow T, Offenbach N (1983) Modification of the initiation of locomotion in Hermissenda: behavioral analysis. Brain Res 271:301-310.

Crow T, Forrester J, Williams M, Waxham N, Neary J (1991) Down regulation of protein kinase $\mathrm{C}$ blocks 5 -HT-induced enhancement in Hermissenda B photoreceptors. Neurosci Lett, in press.

Dixon D, Atwood HL (1989) Conjoint action of phosphatidylinositol and adenylate cyclase systems in serotonin-induced facilitation at the crayfish neuromuscular junction. J Neurophysiol 62:1251-1259.

Farley J, Auerbach S (1986) Protein kinase C activation induces conductance changes in Hermissenda photoreceptors like those seen in associative learning. Nature 319:220-223.

Farley J, Wu R (1989) Serotonin modulation of Hermissenda type B photoreceptor light responses and ionic currents: implications for mechanisms underlying associative learning. Brain Res Bull 22:335351.

Forrester J, Crow T (1988) Protein kinase C inhibition prevents shortterm but not long-term light 5-HT induced enhancement of generator potentials in Hermissenda B-photoreceptors. Soc Neurosci Abstr 14: 839.

Forrester J, Crow T (1989) Kinase inhibitors do not reverse short- or long-term enhancement of light responses in identified Hermissenda B-photoreceptors. Soc Neurosci Abstr 15:1274.

Gelperin A (1981) Synaptic modulation by identified serotonin neurons. In: Serotonin neurotransmission and behavior (Jacobs B, Gelperin A, eds), pp 288-304. Cambridge, MA: MIT Press.

Gershon MD (1977) Biochemistry and physiology of serotonergic transmission. In: Handbook of physiology, The nervous system (Kandel ER, ed), pp 573-623. Bethesda, MD: American Physiological Society.

Goh Y, Alkon DL (1984) Sensory, interneuronal, and motor interactions within Hermissenda visual pathway. J Neurophysiol 52:156169.

Grover LM, Farley J, Auerbach SB (1989) Serotonin involvement during in vitro conditioning of Hermissenda. Brain Res Bull 22:363372.

Hawkins RD (1989) Localization of potential serotonergic facilitator neurons in Aplysia by glyoxylic acid histofluorescence combined with retrograde fluorescent labeling. J Neurosci 9:4214-4226.

Hawkins RD, Schacher S (1989) Identified facilitator neurons L29 and L28 are excited by cutaneous stimuli used in dishabituation, sensitization and classical conditioning of Aplysia. J Neurosci 9:42364245.

Heldman E, Alkon DL (1978) Neurotransmitter synthesis in the nervous system of the mollusc Hermissenda. Comp Biochem Physiol 59: $117-125$

Hodgson TM, Crow T (1987) Light responses of putative motor neurons in the pedal ganglia of Hermissenda are due to synaptic input from photoreceptors. Soc Neurosci Abstr 13:618.

Jacklet JW, Acosta-Urquidi J (1985) Serotonin decreases a background current and increases calcium and calcium-activated current in pedal neurons of Hermissenda. Cell Mol Neurobiol 5:407-412.

Klein M, Kandel ER (1978) Presynaptic modulation of voltage dependent $\mathrm{Ca}^{2+}$ current: mechanism for behavioral sensitization. Proc Natl Acad Sci USA 75:3512-3516.

Land PW, Crow T (1985) Serotonin immunoreactivity in the circumesophageal nervous system of Hermissenda crassicornis. Ncurosci Lett 62:199-205.

Mackey S, Carew TJ (1983) Locomotion in Aplysia: triggering by serotonin and modulation by bag cell extract. J Neurosci 3:14691477.

Mackey SL, Kandel ER, Hawkins RD (1989) Identified serotonergic neurons LCB1 and RCB1 in the cerebral ganglia of Aplysia produce presynaptic facilitation of siphon sensory neurons. J Neurosci 9:42274235.

Neary JT, Naito S, DeWeer A, Alkon DL (1986) $\mathrm{Ca}^{2+}$ /diacylglycerolactivated, phospholipid-dependent protein kinase in the Hermissenda CNS. J Neurochem 47:1405-1411.

Nishizuka Y (1988) The molecular heterogeneity of protein kinase C and its implications for cellular regulation. Nature 334:661-665. 
Ocorr KA, Byrne JH (1985) Membrane responses and changes in cAMP levels in Aplysia sensory neurons produced by serotonin, tryptamine, FMR Famide and small cardioactive peptide ${ }_{\mathrm{B}}\left(\mathrm{SCP}_{\mathrm{B}}\right)$. Neurosci Lett 55:113-118.

Richards WG, Farley J (1987) Motor correlates of phototaxis and associative learning in Hermissenda crassicornis. Brain Res Bull 19: $175-189$.
Sakakibara M, Collin C, Kuzirian H, Alkon DL, Heldman E, Naito S, Lederhendler I (1987) Effects of $\alpha_{2}$-adrenergic agonists and antagonists on photoreceptor membrane currents. J Neurochem 48:405416.

Winer BJ (1962) Statistical principles in experimental design. New York: McGraw-Hill. 